\title{
SUN PROTECTION HABITS AND OCULAR UV DAMAGE OF THE BULGARIAN POPULATION, FIRST RESULTS USING THE METHOD OF CONJUNCTIVAL ULTRAVIOLET FLUORESCENCE
}

\author{
Evgeni Neshkinski ${ }^{1}$, Dobrin Boyadzhiev ${ }^{1,2}$, Christina Grupcheva ${ }^{1,2}$ \\ ${ }^{1}$ Specialised Eye Hospital - Varna \\ ${ }^{2}$ Department of Ophthalmology and Visual Science, Medical University - Varna
}

\begin{abstract}
The purpose of this study is to present our first data and impressions of the sun protection habits and conjunctival ultraviolet autofluorescence (UVAF) of a randomly selected target group.

MATERIALS AND METHODS: A prospective, randomized study, including photographs of 123 subjects (246 eyes) and 200 questionnaires. Subsequently UV photography based on autofluorecence was used. For each eye three pictures were taken - eye in first position, temporal gaze (documenting nasal part of the limbus) and nasal gaze (documenting temporal part of the limbus).

RESULTS: Results are based on the study of 123 subjects (246 eyes). The participants in the study were aged between 5 and 88 years (average age $=39$ ). $87 \%$ of the surveyed believe that there is a risk of UV damage only in the summer. A significant percentage $(55 \%)$ do not pay attention to the special protective equipment such as umbrellas and caps. Photography results were analyzed with specialized software and showed that 83 participants $(67.48 \%)$ have conjunctival autofluorescence, as women prevail (women $n=47$ persons, men $n=36$ persons). The area of conjunctival UVAF tends to increase with age. No correlation between intensive sun exposure (resp. UV radiation) and a sharp increase in the dimensions of the UVAF zone is established, suggesting a chronic damage, in support of which is the demonstrative eye damage in people with high-risk occupations (associated with electric welding, lifeguards and other outdoor occupations).
\end{abstract}

CONCLUSIONS: We suggested that the area and the intensity of UVAF increases with age, and probably corresponds only to a chronic damage. The degree of damage correlates to a large extent to the bad sun protection habits demonstrated in the questionnaire.

Keywords: ophthalmology, autofluorescence, sunlight, ultraviolet radiation, sun protection

Address for correspondence:

Chirstina Nikolova Grupcheva

Specialised Eye Hospital

15 Doyran Str.

Bulgaria, Varna 9002

e-mail: cgrupcheva@gmail.com

Received: November 12, 2014

Accepted: December 19, 2014

\section{INTRODUCTION}

It is well known that the sun is extremely powerful, natural source of UV radiation. Everyone is exposed to ultraviolet radiation from the sun and many artificial sources used in industry, trade and restorative procedures. The sun spectrum includes light, heat and ultraviolet (UV) rays. The ultraviolet range covers a wavelength between 100 and 400 nanometers $(\mathrm{nm})$ and is divided into three parts: UV A, with a wavelength of $320 \mathrm{~nm}$ to $400 \mathrm{~nm}$ which freely pass to the surface of the earth. Although 
they are relatively innocuous, they are a potential cause of severe cumulative damage. UV B is with a wavelength of $280 \mathrm{~nm}$ to $320 \mathrm{~nm}$. About $85 \%$ of them are absorbed by the ozone layer, but the remaining $15 \%$ are harmful to eyes and skin and are a major cause of sunburn. UV C, with a wavelength of $100 \mathrm{~nm}$ to $280 \mathrm{~nm}$, is extremely harmful to humans, but fortunately completely absorbed by the ozone layer approximately $35 \mathrm{~km}$ above the ground (1).

Except the natural UV radiation from the sunlight, numerous sources of artificial UV light, taking place in the modern life, are developed. The field of medicine is no exception - not only for sterilization of instruments and supplies, but also for treatment of different diseases. UV radiation has a proven effect on the suppression or reduction of the inflammatory response in psoriasis (4) and other skin diseases. Circadian rhythm disorders and some psychiatric problems show improvement in symptoms after specific UV treatment (5). UV therapy is indicated also in specific cases of neonatal jaundice (6).

In addition to the purpose of sterilization and treatment, UV light is used in the diagnostics where the Wood's lamp is well known. The principle consists in a long-wave ultraviolet radiation (black light) passing through a filter (Wood's glass), that is blocking all light waves other than those in the range $300-400 \mathrm{~nm}$, with a peak transmission around $365 \mathrm{~nm}$ (7). Fluorescence of tissue is obtained by absorption of light and then emission of radiation with a longer wavelength. Because many substances fluoresce at a specific range, it is used for early diagnosis of a number of surface diseases even before they manifest clinical expression (8).

UV light is very widely spread in nature. It plays an essential role in the presence of all animals. In humans, exposure to UV radiation in small quantities is useful for the normal synthesis of vitamin $\mathrm{D}$ in the body. However, we must assess very carefully the risk-benefit balance, as during prolonged exposure to UV radiation damage to the anterior surface of the eye and the skin may occur. In the long term the excessive exposure to UV light leads to a stimulation of the degenerative changes of the cells in the human body. There are diseases, known in ophthalmology as ophthalmia nivalis (lat. Niphablepsia - snow blindness). It is known that for every for $300 \mathrm{~m}$ altitude, the intensity of UV rays increases by $3-4 \%$. The UV rays are not included in the scope of human perception, so people are not able to feel the harmful effects of excessive exposure. Depending on the strength and intensity of UV radiation damage may occur both in anterior and posterior segment of the eye. Globally, between 12 and 15 million people have disturbances in visual acuity or have completely lost their vision due to cataracts. According to the World Health Organization (WHO) in 20\% of these cases, the main reason is excessive exposure to the harmful effects of UV light (3).

Currently, the UV changes of the eyes are objectively visualized only by using a special lamp developed by Professor Minas Coroneo in Australia $(9,10)$. His unique technology provides clear image of the hyperreflecive zones of the ocular surface believed to be areas of irreversible sun damage.

Based on the current knowledge, technology and experience, the aim of our study was to assess the level of knowledge of the Bulgarian population about the risk of UV damage to the eyes and visualize the changes in the anterior eye surface and try to correlate those.

\section{MATERIALS AND METHODS}

For the purpose of the study a special questionnaire in accessible language that contains appropriate illustrations was developed (Fig. 1).

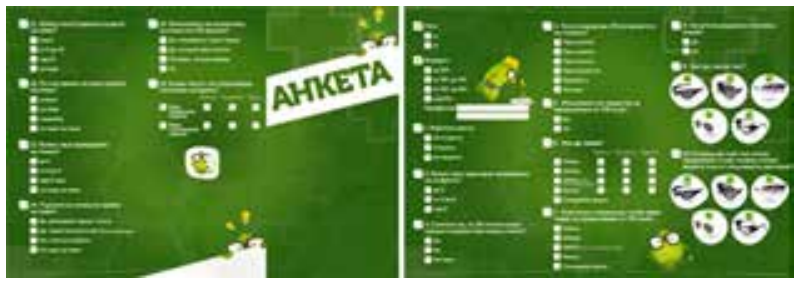

Fig. 1. Questionnaire, consisting of 16 questions and understandable illustrations

The aim was to show the competence on UV protection. The survey included 200 people at an average age 33 years (range 18-63 years). Candidates were not selected on specific criteria (randomized group) as the only condition was to be Caucasian Bulgarian citizens. The questionnaire was designed as such to extract knowledge: whether they think that excessive exposure to sunlight can lead to 
damage to their eyes and is there seasonality of UV rays; whether they use any protection and if yes what exact type and modality; is there a correlation between the age and the studied areas of damage and a link between the changes on the anterior eye surface and protective habits.

The objective part of the study was conducted with ultraviolet fluorescence photography (UVFP) system, provided by Vision Care Institute Prague. The device consists of height adjustable table, chinstrap for fixing the head, unit on which the photographic system was mounted and allowed focusing and setting in all of the three axes, digital SLR photography system with a macro lens, two flashes, located under a constant angle to the eye of the participant and fixation mark enabling each photo to be made under the same angle for repeatability and comparability of the results. The used setup is identical to the used in the study of UVAF distribution of Norfolk Island $(12,13)$. UV-induced fluorescence was achieved with two electronic flashes, equipped with a UV pass filter between 300 and $400 \mathrm{~nm}$, with peak transmittance in the range of $\lambda_{\text {max }}=365 \mathrm{~nm}$. To clear the image and document primarily autofluorescencent structures, an additional UV barrier filter (with a corresponding range) and infrared filter (to eliminate the red color) were installed. The setting allowed verification of the quality of each image in real time, thus low quality photos were deleted and taken again. Photo recording was in the largest format and resolution, at a magnification of $0.94 \mathrm{X}$. For the purpose, native images (control) with a natural reflected light were made first, and then using UV photography system, three photos were taken of each eye - one in first position (central) and two with gaze directed about $35^{\circ}$ from the optical axis of the camera (nasal and temporal). Despite the use of filters, some images got unwanted red reflex, which if necessary could be eliminated with the removal of the red channel in a specialized software (equivalent to the use of cyan filter in a photographic system (14)), but in most cases had no impact on the quality of pictures and analysis of the results.

The collected data were analyzed and processed statistically (SPSS). In cases where the photos required image processing, image software was used $(14,15)$.

\section{RESULTS}

The survey included 123 persons (246 eyes). From the entire group, 61 people $(49.59 \%)$ were men and $62(50.41 \%)$ - women. The youngest participant was 5 years old, and the oldest - 88 years old. The average age of the participants was 39 years. In the interest of further processing of the data, subjects were divided into four groups (Fig. 2):

1. First group - under 25 years old $-\mathrm{n}=13$ participants $(10.56 \%)$

2. Second group $-26-40$ years old $-\mathrm{n}=61$ participants $(49.59 \%)$

3. Third group $-41-60$ years old. $-\mathrm{n}=29$ participants (23.57\%)

4. Fourth group - over 61 years old $-\mathrm{n}=20$ participants (16.26\%).

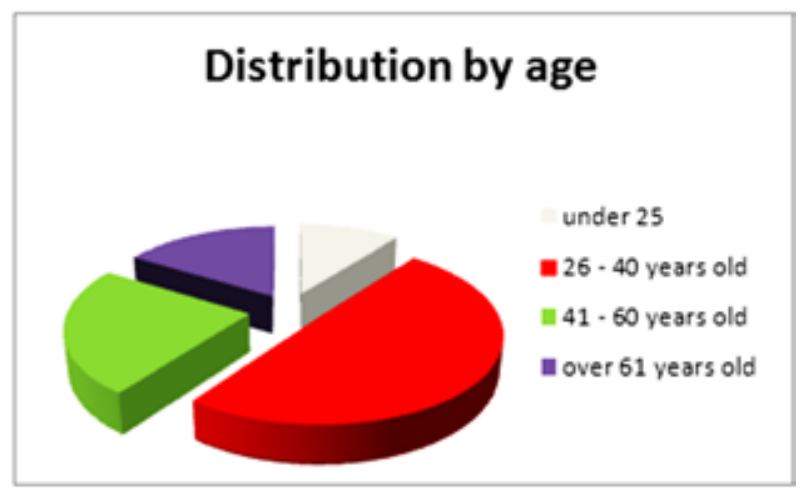

Fig. 2. Distribution by age highlighting prevalence of young participants

Throughout the whole target group of 123 people, in 83 people $(67,48 \%)$ we found evidence of conjunctival UVAF. Females were more affected by the changes (average 56, 63\% - $n=47$ persons) (Fig. 3).

The differences between both eyes of the participants, and a difference between the areas of autofluorescence nasal and temporal of the limbus were analyzed. In 40 of the eyes with presence of UVAF (24.1\%) the changes were more pronounced nasal from limb. In 80 eyes with UVAF (48.20\%) the zones of autofluorescence were the same size and intensity in the nasal and temporal portion of the conjunctiva and in the remaining 46 eyes $(27.70 \%)$ the areas were more pronounced temporal of the limbus (Fig. 4). No significant difference in the degree of damage between the two eyes of the participants was found. 


\section{Distribution by gender}

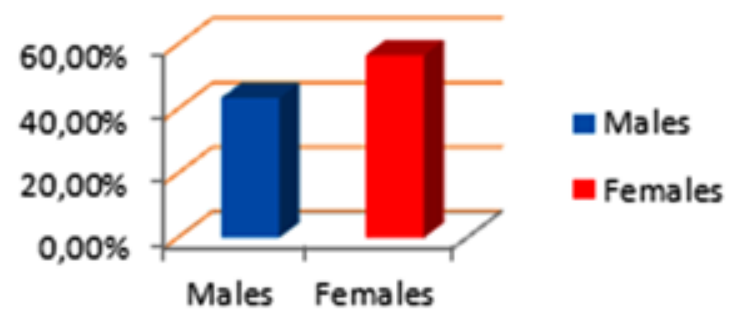

Fig. 3. Distribution of UVAF presence by gender

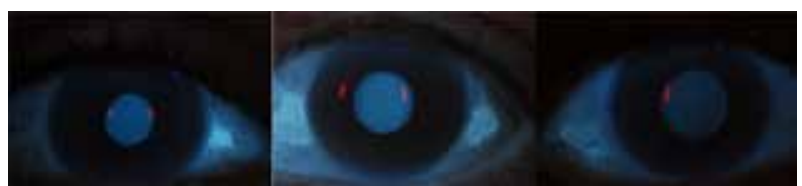

Fig. 4. Difference between the area and intensity of UVAF in different participants

From the analysis of the data compared to the age of the subjects, we obtained the following results (Tabl. 1):

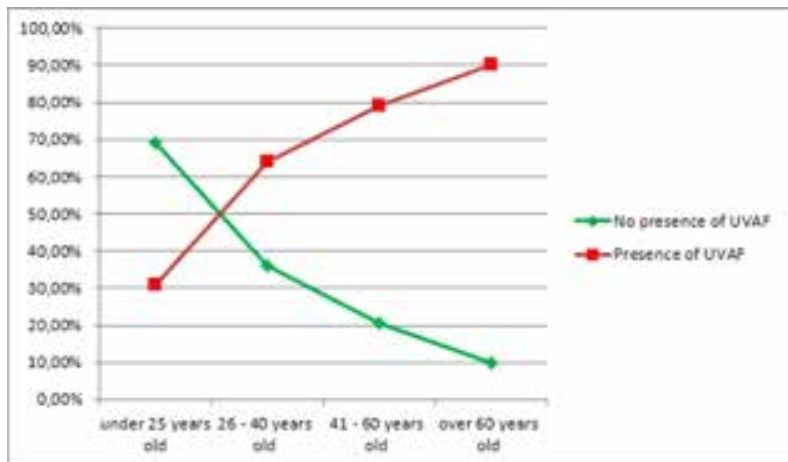

Fig. 5. Correlation between increasing age and presence of UVAF

obtained using the UV camera. Finally, a comparison between the data obtained from the questionnaire and damage on the front surface of respondents was made.

It was found that $87 \%$ of the surveyed believe that there is a risk of UV damage only in the summer. Most of them think that the protective sunglasses should be worn only in the summer, underestimating the harmful effects of the sun in winter. A significant percentage (55\%) do not pay attention to the special protective equipment such as umbrellas and caps. $76 \%$ of respondents, who wear sunglasses, do not consider the relationship between the shape

Tabl. 1. Distribution by age and presence of UVAF

\begin{tabular}{|c|c|c|c|}
\hline & Total & No presence of UVAF & With presence of UVAF \\
\hline Under 25 years old & $\mathrm{n}=13$ & $\begin{array}{c}\mathrm{n}=9 \\
(69.23 \%)\end{array}$ & $\begin{array}{c}\mathrm{n}=4 \\
(30.77 \%)\end{array}$ \\
\hline $26-40$ years old & $\mathrm{n}=61$ & $\begin{array}{c}n=22 \\
(36.06 \%)\end{array}$ & $\begin{array}{c}n=39 \\
(63.94 \%)\end{array}$ \\
\hline $41-60$ years old & $\mathrm{n}=29$ & $\begin{array}{c}n=6 \\
(20.70 \%)\end{array}$ & $\begin{array}{c}\mathrm{n}=23 \\
(79.30)\end{array}$ \\
\hline Over 60 years old & $\mathrm{n}=20$ & $\begin{array}{c}n=2 \\
(10.00 \%)\end{array}$ & $\begin{array}{c}n=18 \\
(90.00 \%)\end{array}$ \\
\hline
\end{tabular}

Represented graphically, these results indicate a correlation, that as the age of the population increases, so does the percentage of participants with presence of UVAF zones and the intensity of the changes in each of them (Fig. 5).

\section{DISCUSSION}

The first part of the study represents the degree of competence of the people on the protection from $\mathrm{UV}$ rays and the second is an analysis of the results of the glasses and their degree of protection. Many of the participants agree that additional information and education about UV damage is needed.

Throughout the whole target group of 123 people, in 83 people $(67.48 \%)$ we found evidence of conjunctival UVAF. Such data shows slightly higher percentage than the one, obtained in another similar study (62\% presence of UVAF (11)). Females are more affected by the changes (average $56.63 \%, n=47$ per- 
Sun protection habits and ocular uv damage of the Bulgarian population, first results using the method of conjunctival ...

sons), whereas for men the corresponding percentage is $43.37 \%, \mathrm{n}=36$ persons.

In our study we encountered that the percentage of participants with presence of UVAF zones and the intensity of the changes at each of them depend on age. This finding contrasts the study of Norfolk Island, which results show that UVAF is more expressed in young people and men (12). The resulting differences between the two studies could be explained by the different geographical location of the study population, sun protection habits, diet, etc. Differences in genetic background could also affect the results (13).

It is clear that habits for eye protection from the harmful effect of the UV light are missing in this study among the Bulgarian population, but most probably that is valid for the entire European population. Somehow, in Europe we believe that the sun is damaging only during the summer season and therefore, if any care is taken, that is connected to the outdoor summer time. Our study is a pilot one but proved that there are cumulative effects of UV exposure on the anterior ocular surface. How significant is the fluorescence of the exposed conjunctiva is still to be investigated, but most probably it is associated with surface changes, damage of the tear film and therefore long term decreased quality of life. The role of the eye care practitioner is to increase public awareness of all year round possibility of UV damage and the knowledge about possible prophylactic measures.

\section{CONCLUSION}

This paper delivers results from a large, population-based study of conjunctival UVAF, which is based on the thesis that UVAF is an objective marker of damage to the anterior ocular surface, and the degree of competence on sun protection habits. Our results show that with increasing age the areas of UVAF increase both in size and intensity. These results contrast with other studies carried out in a similar way $(12,13)$. This requires further analysis of the reasons for the differences. Another conclusion that can be derived is that the degree of damage correlates to a large extent to bad protection habits demonstrated in the questionnaire. No significant difference was found between the damages in the two eyes of the same person.
Future studies and additional analysis of the results will establish a link between the documented changes, sun exposure time, environment, and social habits of the Europeans. The significance of the autofluorecent areas also needs further investigation.

\section{REFERENCES}

1. ISO 21348 Definitions of Solar Irradiance Spectral Categories.

2. Buron N, Micheau O, Cathelin E et al. Differential mechanisms of conjunctival cell death induction by ultraviolet irradiation and benzalkonium chloride. Inv Ophthalmol Vis Sci, 2006;47(10):4221-30.

3. WHO Library. Global Solar UV Index: A Practical Guide. ISBN 9241590076

4. „UVB Phototherapy“ (php). National Psoriasis Foundation, USA. Archived from the original on 22 June 2007. Retrieved 2007-09-23 05;11:227-56.

5. Lam RW, Buchanan A, Mador JA, Corral MR, Remick RA, The effects of ultraviolet-A wavelengths in light therapy for seasonal depression, Journal of Affective Disorders, 1992;24(4):237-243.

6. Maisels JM, McDonagh AF. Phototherapy for Neonatal Jaundice. N Engl J Med, 2008;358:920-928.

7. David Ponka, MD CM CCFP(EM) FCFP, Wood lamp examination. Can Fam Physician, Sep 2012;58(9): 976.

8. Monici M. Cell and tissue autofluorescence research and diagnostic applications. Biotechnol Annu Rev, 2005;11:227-56. Review.

9. Ooi JL, Sharma NS, Papalkar D, Sharma S, Oakey $\mathrm{M}, \mathrm{Dawes} \mathrm{P}$ et al. Ultraviolet fluorescence photography to detect early sun damage in the eyes of school-aged children. Am J Ophthalmol 2006;141: 294-298.

10. Ooi JL, Sharma NS, Sharma S, Papalkar D, Oakey M,Dawes P et al. Ultraviolet fluorescence photography:patterns in established pterygia. Am J Ophthalmol, 2007;143:97-101.

11. Wolffsohn JS, Drew T, Sulley A. Conjunctival UV autofluorescence--prevalence and risk factors. Cont Lens Anterior Eye, 2014 Dec;37(6):427-30.

12. Sherwin JC, Hewitt AW, Kearns LS et al. The association between pterygium and conjunctival ultraviolet autofluorescence: TheNorfolk Island Eye Study. Acta Ophthalmologica, 2013;91:363-370. 
13. JC Sherwin, AW Hewitt, LS Kearns, MT Coroneo, LR Griffiths and DA Mackey. Distribution of conjunctival ultraviolet autoflourescence in a population-based study: the Norfolk Island Eye Study. Eye, 2011;25:893-900.

14. Schneider CA, Rasband WS, Eliceiri KW. NIH Image to ImageJ: 25 years of image analysis. Nat Methods, 2012 Jul;9(7):671-5.

15. Collins TJ. ImageJ for microscopy. Biotechniques, 2007 Jul;43(1 Suppl):25-30. 